follows: impaired glucose tolerance (19\%) and CF-associated diabetes mellitus (43\%). Calculated indices of insulin resistance (Homa and Caro) showed that $71 \%$ of patients with carbohydrate metabolism disorders had a decrease in Caro $(<0,332)$ on the $120^{\text {th }}$ minute of the oral glucose tolerance test, which indicated insulin resistance.

The average HbA1c levels in children with $\mathrm{CF}$-associated diabetes mellitus were $7,3 \pm 3,4 \%$, children with glucose tolerance $-6,3 \pm 0,4 \%$ and in children with normal carbohydrate metabolism - 5,8 $\pm 0,3 \%$.

The CGMS assessment detected hyperglycemia in $81 \%$ patients, while carbohydrate metabolism disorders were diagnosed only in $28 \%$ of the cases. Stable postprandial hyperglycemia was detected with CGMS in $91 \%$ children aged $\geq 11$ and $64 \%$ in group 3-11.

The 74,4\% patients had delF508 mutations (homozygous$32 \%)$. All the cases of CF-associated diabetes mellitus were diagnosed in these patients.

Conclusions The incidence of carbohydrate metabolism disorders in CF-children was reliably higher than in overall population (28\%). Carbohydrate metabolism disorders and CFassociated diabetes were reliably more frequent in children over 11 years of age.

Insulin resistance must be the most likely cause of carbohydrate metabolism disorders.

CGMS in patients with cystic fibrosis helps to detect hidden hyperglycemia that cannot be detected with the help of standard methods.

Carbohydrate metabolism disorders and CF-associated diabetes mellitus are more frequent in patients with delF508 mutation.

\section{OC45 POLYCYSTIC OVARIAN SYNDROME IN ADOLESCENTS: UTILISING DISCOVERY PROTEOMICS TO IDENTIFY NOVEL NON-INVASIVE BIOMARKERS}

\begin{abstract}
1,2,3,4 Harriet Gunn*, 3,4Vhari Forsyth, ${ }^{2}$ Russell Viner, ${ }^{1}$ Kevin Mills, ${ }^{3,4}$ Katharine Steinbeck. ${ }^{1}$ Translational Mass Spectrometry Research Group, UCL Great Ormond Street Institute of Child Health, London, UK; ${ }^{2}$ Population, Policy and Practice Programme, UCL Institute of Child Health, London, UK; ${ }^{3}$ Academic Department of Adolescent Medicine, Sydney Children's Hospital Network, Sydney, Australia; ${ }^{4}$ Discipline of Child and Adolescent Health, The University of Sydney, Sydney, Australia
\end{abstract}

\subsection{6/archdischild-2019-epa.43}

Background Polycystic ovarian syndrome (PCOS) is the most common hormone disorder in females, affecting 4-20\% of the population. PCOS is associated with metabolic dysfunction, pro-inflammation and mood disorders. Despite this, it is poorly understood, and diagnosis and management remain challenging in adolescents. Proteomics enables a better understanding of disease mechanisms and facilitates the identification of novel biomarkers.

Aims

1. To better understand the clinical phenotype of PCOS in adolescents.

2. To undertake discovery proteomic urine profiling using ultra-performance liquid chromatography-mass spectrometry (UPLC-MS/MS) to identify novel non-invasive biomarkers of PCOS.

Method In this prospective longitudinal study, females aged 12-19 years meeting NIH diagnostic criteria for PCOS were recruited from adolescent endocrine and gynaecology clinics. At baseline and annual follow-up, the following were measured: pituitary, adrenal and ovarian hormones, anti-Müllerian hormone, inflammatory and metabolic markers including an oral glucose tolerance test, psychometric questionnaires, menstrual records, pubertal assessment, anthropometric parameters and pelvic ultrasounds. We have undertaken UPLC-MS/MS and developed new methods for discovery proteomic profiling of urine samples in an attempt to identify new disease mechanisms, drug targets and potential biomarkers.

Results To date, 37 participants have been recruited (median age 15.0 years, range 12.6-18.3), and 22 have completed annual follow-up. Clinical signs at presentation included acne (89\%), hirsutism (78\%), acanthosis nigricans (49\%) and overweight/obesity (81\%). Two-thirds of participants had depressive or anxiety symptoms. Only one-third were known to mental health services. Metabolic dysfunction was common; elevated body fat (88\%), dyslipidaemia (24\%), insulin resistance (62\%), and impaired fasting glucose, impaired glucose tolerance or type 2 diabetes (40\%). AMH was elevated in one-third of participants and three-quarters had an elevated free androgen index. Elevated inflammatory markers (CRP/ESR) were present in $40 \%$ participants. Only three participants had definitive ultrasonographic evidence of PCOS. Interventions included lifestyle advice only (27\%), combined oral contraceptive pill (COCP) \pm anti-androgen (16\%), metformin (30\%) or metformin + COCP \pm anti-androgen $(27 \%)$.

Conclusion and Future Directions Diagnosing PCOS in adolescents remains challenging; acne and irregular menstrual cycles are common and ultrasonographic diagnosis of PCOS is suboptimal. Given the high prevalence of metabolic and mental health disorders, early diagnosis and intervention are imperative. We describe the use of urinary proteomics to study metabolic pathways affected in PCOS and the potential identification of novel non-invasive biomarkers. Subsequently, we will use this hypothesis-generating data-set to create a non-invasive and clinically translatable assay to aid diagnosis and stratify management of this common adolescent condition.

\section{OC46 THE POPULATION INCIDENCE OF CHILDHOOD GONADOBLASTOMA OVER 20 YEARS IN THE REPUBLIC OF IRELAND}

${ }^{1}$ Aisling R Geoghegan*, ${ }^{2}$ Sally Ann Lynch, ${ }^{3}$ Ciara M McDonnell, ${ }^{4}$ Michael McDermott, ${ }^{5}$ Feargal Quinn, 'Declan Cody, 'Susan M O'Connell. 'Endocrinology Department, Our Lady's Children's Hospital Crumlin, Dublin, Ireland; 'Genetics Department, Our Lady's Children's Hospital Crumlin, Dublin, Ireland; ' ${ }^{3}$ Endocrinology Department, Tallaght University Hospital, Dublin, Ireland; ${ }^{4}$ Histopathology Department, Our Lady's Children's Hospital Crumlin, Dublin, Ireland; ${ }^{5}$ Paediatric Surgery, Our Lady's Children's Hospital Crumlin, Dublin, Ireland

\subsection{6/archdischild-2019-epa.44}

Background Gonadoblastoma (GB) is a rare tumour of the gonads presenting in childhood or adolescence. It is a lesion composed of a mixture of germ cells at different stages of maturation, with low malignant potential. It is associated with disorders of sex development, most commonly Turner mosaic syndrome with $\mathrm{Y}$ chromosome material (TMSY), and 46XY gonadal dysgenesis (GD). Little is known about the natural history and incidence, however prophylactic gonadectomy is recommended.

Objectives To determine the incidence and clinical features of GB presenting in childhood in the Irish Republic (RoI) from 1999-2018 inclusive.

Methods A retrospective review of children and adolescents with a diagnosis of GB was undertaken using the records of the National Cancer Registry Ireland (NCRI) and the 